\title{
Reduced Graphene Oxide Thin Films with Very Large Charge Carrier Mobility Using Pulsed Laser Deposition
}

\author{
A Bhaumik ${ }^{1,2}$, A Haque ${ }^{1,2 *}$, MFN Taufique ${ }^{1,3}$, P Karnati ${ }^{1,4}$, R Patel $^{1}$, M Nath5 and K Ghosh ${ }^{1}$ \\ ${ }^{1}$ Department of Physics, Astronomy and Materials Science and Center for Applied Science and Engineering, Missouri State University, Springfield, MO 65897, USA \\ ${ }^{2}$ Department of Materials Science and Engineering, North Carolina State University, Raleigh, NC 27695, USA \\ ${ }^{3}$ School of Mechanical and Materials Engineering, Washington State University, Pullman, WA 99164-2920, USA \\ ${ }^{4}$ Department of Materials Science and Engineering, Ohio State University, Columbus, OH, 43210, USA \\ ${ }^{5}$ Department of Chemistry, Missouri University of Science and Technology, Rolla, MO 65409, USA
}

\begin{abstract}
Large area reduced graphene oxide (RGO) thin films have been grown using pulsed laser deposition (PLD) technique. A very large carrier mobility of $372 \mathrm{~cm}^{2} \mathrm{~V}^{-1} \mathrm{~s}^{-1}$ has been observed in a PLD grown RGO thin film with a large $s p^{2}$ carbon fraction of $87 \%$ along with narrow Raman $2 D$ peak profile. The fraction of $s p^{2}$ carbon and carbon/oxygen ratios are tuned through PLD growth parameters, and these are estimated from X-ray photoelectron spectroscopy (XPS) data. The electrical properties of the RGO thin films are comprehended by the intensity ratios between different optical phonon vibrational modes of Raman Spectra. The photoluminescence spectra also indicate a less intense and broader blue fluorescence spectrum detecting the presence of miniature sized $s p^{2}$ domains in the near vicinity of $\pi^{*}$ electronic states which favor the variable range hopping transport phenomena. This study on large area RGO thin films with very large carrier mobility fabricated by PLD process will be very useful for high mobility electronic device applications and could open a roadmap for further extensive research in functionalized 2D materials.
\end{abstract}

Keywords: Reduced graphene oxide; Raman spectroscopy; Variable Range Hopping; 2D-materials; Pulsed laser deposition

\section{Introduction}

Chemical functionalization of graphene has enticed significant research interests due to its potential in obtaining an optical band gap and subsequent tuning of its optoelectronic properties for device applications [1,2]. The solution processed route for producing RGO flakes has received significant attention because of its high throughput manufacturing, and tunable electrical and optical properties by controlling the ratio of $s p^{2}$ and $s p^{3}$ hybridized carbon clusters $[3,4]$. Functionalization of graphene that creates disorders and electron localization-hopping phenomena plays a significant role in determining its physical properties $[5,6]$. However, the electronic parameters for device applications are not quite good, for example, the carrier mobility of solution processed RGO is not high enough for many device applications [6]. The change in physical properties of functionalized graphene is due to (i) the transformation of hybridization state in carbon atoms, (ii) formation of a barrier (scattering entity) at the functionalization site and within the electron potential sequence, (iii) distortion of its two-dimensional planar lattice due to functionalization or thermal energy, and (iv) introduction of $s p^{2}$ clusters and defects thereby introducing different energy levels [7]. The alterations in the chemical, structural, and electronic properties of RGO can be originated by the molecular level interaction arising by a number of phenomena i.e. (i) covalent bonding, (2) $\pi-\pi^{*}$ interfacing, (3) lattice substitution, or (4) physisorption [8]. In graphene the direct covalent functionalization of $s p^{2}$ hybridized carbon transforms it into a tetrahedral $s p^{3}$ hybridized carbon. This conversion of the hybridization state of $\mathrm{C}$ atoms causes a loss of the free, $s p^{2}$ associated $\pi$ electrons. On the contrary, the substitution of carbon atoms with other elements (for instance $\mathrm{N}$ or $\mathrm{B}$ ) retains the $s p^{2}$ character disrupting the $\pi$-cloud continuum [9]. Additionally, it is imperative to remark that the non-covalent functionalization of graphene via $\pi$ - $\pi^{*}$ interfacing or physisorption does not distort the $s p^{2}$ network. However this phenomena changes the doping concentration, raises the density of electron-hole puddles substantially, and also may create scattering sites for the free charge carriers [9].
The most studied approach of graphene functionalization is covalent functionalization which has been conducted by following different routes. This process causes for the significant changes to the electrical properties in graphene structure. Reducing GO produces RGO; however, RGO contains unreduced, covalently bonded oxy groups [10]. The removal of the $\pi$ electron from the carbon atom reduces the carrier density and can introduce an optical band gap. The functionalizing molecule can also introduce energy levels (edge states and functionalization states) in the band structure of covalently functionalized graphene (CFG) to make it an n-type or p-type semiconductor [9]. Therefore, a combination of the carrier deficiency at the $s p^{3}$ site, the associated disruption of the electron-potential continuum, and the distorted planar lattice cause a drastic reduction in charge carrier mobility and a change in charge polarity in graphene. Compare to graphene (mobility of $10,000-50,000 \mathrm{~cm}^{2} \mathrm{~V}^{-1} \mathrm{~s}^{-1}$ and intrinsic mobility limit $200,000 \mathrm{~cm}^{2} \mathrm{~V}^{-1} \mathrm{~s}^{-1}$ at room temperature), RGO, with few covalently bonded residual functional groups, exhibits a drastically reduced carrier mobility $\left(0.05-200 \mathrm{~cm}^{2} \mathrm{~V}^{-1} \mathrm{~s}^{-1}\right)$, p-type conductivity, and a finite effective optical band gap of 0.2 to $2 \mathrm{eV}$ [5]. However a recent report indicates that the FET mobility in RGO can be increased up to $\sim 1000 \mathrm{~cm}^{2} \mathrm{~V}^{-1} \mathrm{~s}^{-1}$ by microwave reduction technique [11]. Till date, the most common approach to synthesize RGO involved multistep processing i.e. (i) synthesizing graphene oxide (GO) by oxidative exfoliation of graphite (Hummers' method) and (ii) reducing GO by

*Corresponding author: Ariful Haque, Department of Physics, Astronomy and Materials Science and Center for Applied Science and Engineering, Missouri State University, Springfield, MO 65897, USA, Tel: (313) 413-6681; E-mail: ahaque@ncsu.edu

Received July 26, 2017; Accepted July 31, 2017; Published August 11, 2017

Citation: Bhaumik A, Haque A, Taufique MFN, Karnati P, Patel R, et al. (2017) Reduced Graphene Oxide Thin Films with Very Large Charge Carrier Mobility Using Pulsed Laser Deposition. J Material Sci Eng 6: 364. doi: 10.4172/21690022.1000364

Copyright: (c) 2017 Bhaumik A, et al. This is an open-access article distributed under the terms of the Creative Commons Attribution License, which permits unrestricted use, distribution, and reproduction in any medium, provided the original author and source are credited. 
thermal and chemical processes. To remove additional oxidative agents in the GO structure, centrifugation, filtration, etc. are required, which increases the production cost of large area GO. Successive reductions of the cleaned GO using additional treatments are also required to produce high quality RGO which usually involves more processing steps such as thermal reduction, photocatalytic reduction, chemical reduction, etc. $[12,13]$. These processing steps increase complexity in RGO synthesis.

PLD has been established as a very efficient technique to produce thin films of various materials with unique properties. PLD has a number of advantages over other physical vapor deposition techniques. For example, PLD can transfer the composition of the target to the substrate. Since the plume temperature in PLD is very high (order of $10^{4} \mathrm{~K}$ ) it gains more energy and increases the surface mobility which leads to improved quality films. Many materials can be deposited using a wide variety of reactive gases such as oxygen, nitrogen, and other gas mixture. This is possible because there are no ions or evaporation sources that contain hot filament in the vacuum. Because of the many advantages, PLD has been extensively used for the growth of High TC oxides, ferroelectrics, ferrites, semiconductors, polymers, metals, alloys, carbon nanotubes, and many others [11-13]. However, to our knowledge there is no report available on the growth of highquality graphene and RGO films using PLD [14]. This study attempts to synthesize high-quality large area RGO thin films with tunable electronic and optical properties using PLD.

By employing PLD we have fabricated RGO thin films with controlled variation in $s p^{2} / s p^{3}$ ratios calculated from XPS analysis, and FWHM of $2 D$ peak along with $I 2 D / I G$ ratios obtained from Raman spectroscopy. These factors interplay significant roles to influence the electronic properties of thin films of RGO. The ratios of $s p^{2} / s p^{3}$, hence, structural and electronic properties can be controlled through growth parameters of the PLD and introducing a seed layer of single layer of graphene on a substrate before the growth of RGO. Here we present results of some representative samples termed as sample A, B, C, and $\mathrm{D}$ on the basis of the gradual increment in the intensity and sharpness of the $2 D$ peak. Sample D shows significantly high concentration of $s p^{2} / s p^{3}$ ratio, has sharpest $2 \mathrm{D}$ peak compared to the other samples and exhibits best electronic properties. To better understand the improved electronic transport properties of PLD grown RGO samples detailed temperature dependent electronic transport properties have also been investigated and correlated with structural and optical properties.

\section{Experimental Section}

Thin films of RGO were deposited on $\mathrm{SiO} 2(300 \mathrm{~nm}) / \mathrm{Si}$ substrates purchased from MTI corporation by pulsed laser deposition (Excel Instrument, PLD-STD-18) using a dense and high-quality graphite target (99.9\%) purchased from Kurt J. Leskar. A KrF excimer laser (Lambda Physik, COMPEX 201) with energy density of $2 \mathrm{~J} \mathrm{~cm}^{-2}, \lambda=248$ $\mathrm{nm}$, pulsed duration of $20 \mathrm{~ns}$, was used at a pulse rate of $10 \mathrm{~Hz}$ for the deposition. Thin films were grown by varying the number of PLD shots (sample A: 5000 shots, sample B: 100 shots, sample C: 10,000 shots, and sample D: 500 shots) maintaining a constant oxygen pressure $\left(1 \times 10^{-5}\right.$ Torr) and growth temperature $\left(700^{\circ} \mathrm{C}\right)$ in the PLD chamber. Before the growth of sample $\mathrm{D}$, a seed layer of a single layer graphene was introduced to improve the mobility of the samples. All the deposited films were cooled in forming gas, a gas mixture of $\mathrm{Ar}(96 \%)$ and $\mathrm{H} 2$ (4\%) at pressure of $10^{-4}$ Torr. The number of laser shots were critically determined for the best growth of reduced graphene oxide thin films.

To calculate the fraction of GO and RGO and lattice parameters of these two phases, X-ray diffraction (XRD) measurement was carried out. XRD data of all thin film samples were recorded using a X-ray diffractometer (Bruker, D8 Discover) using the theta-2theta scan with $\mathrm{CuKa}(\lambda=1.5405 \AA)$. XRD data were analyzed using GaussianLorentzian fitting profile using Origin Pro 8.5.1 software. The vibrational phonon modes were determined by Raman spectroscopy using 532nm green laser (Horiba, Labram Raman-PL). Photoluminescence (PL) measurements were carried out employing $325 \mathrm{~nm}$ UV laser using the above-mentioned instrument. The Raman spectra and PL spectra was analyzed using Gaussian and Lorentzian peak fittings in NSG Lab spec software. The ratio of $s p^{2} / s p^{3}$ carbon and carbon/ oxygen ratio were estimated from X-ray photoelectron spectroscopy (XPS) performed on a Kratos Axis 165 photoelectron spectrometer with a hemispherical 8 channel analyzer where photoemission was stimulated by a monochromatic $\mathrm{Al}$ source. The electrical conductance and Hall coefficient measurements were carried out by a standard four-probe technique. The Ohmic contacts were made using lowtemperature In-Ag solder. The sample voltage was measured with a nanovoltmeter (Keithley, 182) with a current of 1 to $100 \mathrm{~mA}$ using a $20 \mathrm{ppm}$ stable current source (Keithley, 220). The magnetic field dependence of the Hall effect was measured with the field applied perpendicular to film surface in the Van der Pauw configuration. The low-temperature resistance measurements were carried out using a SQUID magnetometer coupled with Labview programming interface. The temperature was stabilized within $0.1 \%$ of that temperature and measured using a calibrated sensor. The morphology of all RGO thin film samples was characterized using atomic force microscopy (AFM). AFM imaging was performed under ambient conditions using a Digital Instruments (Veeco) Dimension-3100 unit with Nanoscope III controller, operated in tapping mode. The etched silicon tapping tips employed in the study had a nominal apex radius of curvature of $\sim 10-20 \mathrm{~nm}$ and a cone angle of $\sim 34^{\circ}$. The spring constant of the cantilever was $\sim 42 \mathrm{~N} / \mathrm{m}$. The cantilevered tip was oscillated close to the mechanical resonance frequency of the cantilever (typically, 200-300 $\mathrm{kHz}$ ) with amplitudes ranging from $\sim 10$ to $30 \mathrm{~nm}$.

\section{Results and Discussion}

\section{Raman scattering}

Figure 1 represents the room temperature unpolarized Raman spectroscopy data of RGO thin films deposited onto $\mathrm{SiO} 2(300 \mathrm{~nm}) /$ Si. Raman spectra of graphene-based materials show almost all the significant features in the range between $1000 \mathrm{~cm}^{-1}$ to $3000 \mathrm{~cm}^{-1}$, and the corresponding Raman modes are known as $D, G, 2 D$ and $D+G$ band [15]. To ensure the best fit an extra peak was found in between $D$ and $G$ band which is known as $f$ band [16]. The Alg symmetry phonon near K zone boundary involved with the breathing modes of $s p^{2}$ carbon atoms present in graphene and GO introduces $D$-peak around 1355 $\mathrm{cm}^{-1}$ which arises only due to the presence of defects and asymmetry in the lattice [17]. The $D$ peak position of the as-synthesized samples are in between $1345 \mathrm{~cm}^{-1}$ to $1350 \mathrm{~cm}^{-1}$. The E2g phonon mode in the Brillouin zone center correspond to the $G$ peak is positioned in between 1500 $\mathrm{cm}^{-1}-1630 \mathrm{~cm}^{-1}$. The origin of the $G$ peak is due to the bond stretching of $s p^{2} \mathrm{C}$ atoms both for rings and chains [17]. It has been observed that the $G$ peak of RGO is shifted to lower frequencies due to the removal of functional groups by reduction reaction [18]. Stiffening of the $G$ mode also indicates the increase in carrier density of either sign [19]. The $G$ peak position of the four samples observed in between $1597-1600 \mathrm{~cm}^{-1}$ indicates the presence of RGO structure on the thin films.

The ratio between the peak intensities of $D$ band and $G$ band is an 

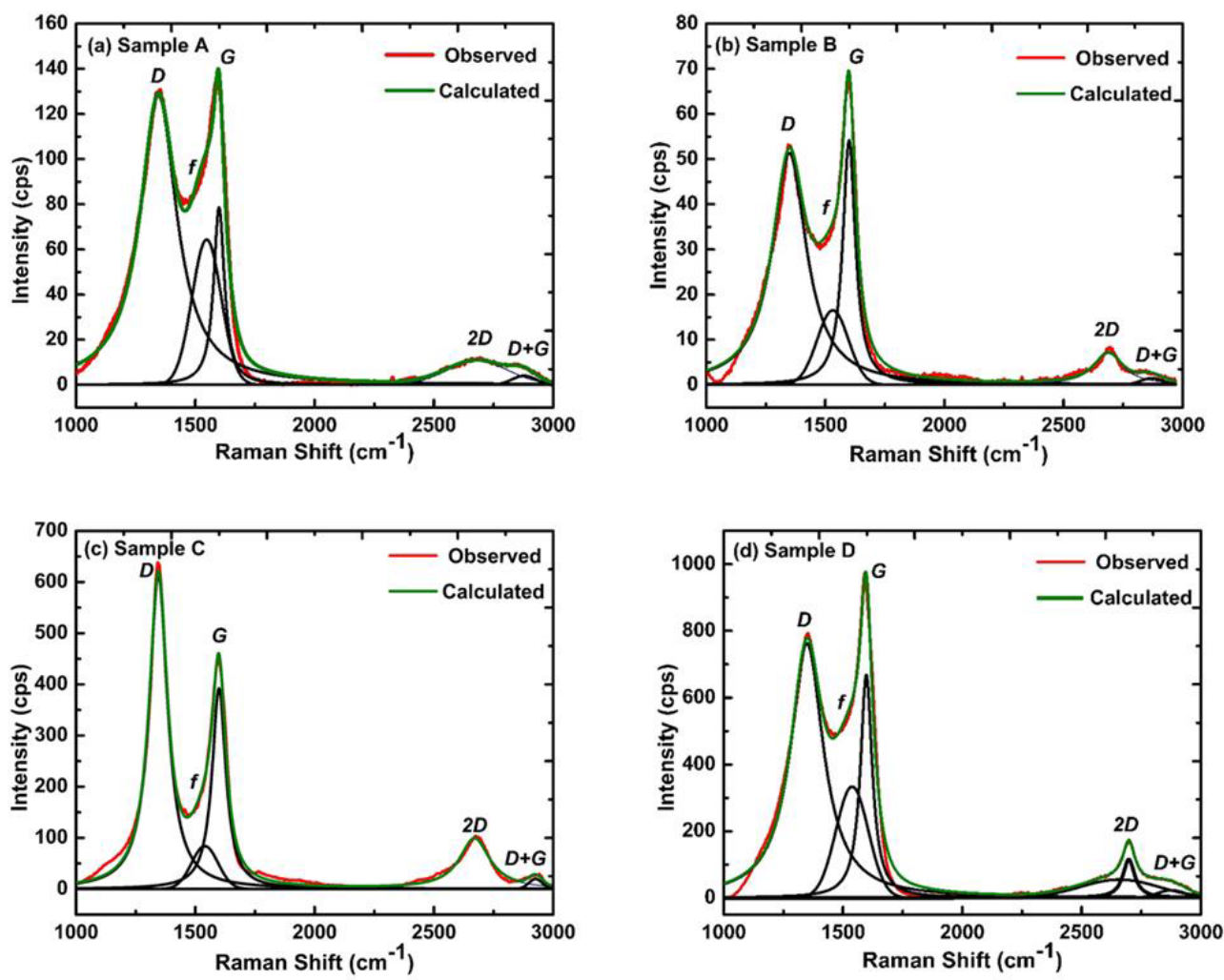

Figure 1: Raman spectra of RGO thin film samples synthesized by PLD technique, (a) Sample A, (b) sample B, (c) sample C, and (d) sample D, showing the variations in FWHM of $2 \mathrm{D}$ peaks and I2D/IG, I2D/ID+G, and ID/IG, ratios to illustrate the restoration of sp2 carbon clusters and the degree of reduction.

indication of the degree of reduction [20]. Researchers suggested that according to Tuinstra and Koenig (TK) relationship with increasing the disorder in the structure, $I D / I G$ increases. However, further reduction reaction creates more disorder and distortion on the $s p^{2}$ structure [17]. As a result, $I D$ decreases compared to $I G$ and TK relationship no longer exists. The corresponding $I D / I G$ ratio of sample A, B, C and D is 1.653 , $0.973,1.565$, and 1.14 , respectively. The higher $I D / I G$ ratio signifies that higher growth temperature and reaction environment assist in reduction of the GO thin films.

The second order overtone of the $D$ peak around $2710 \mathrm{~cm}^{-1}$ is known as $2 D$ peak. This peak is the characteristic to the double resonance transition resulting from the generation of two phonons with opposite momentum to each other $[21,22]$. It has been reported that the $2 D$ peak profile is sharp in the Raman spectrum of Graphene, while small $2 D$ peak intensity compared to $D$ and $G$ peaks is an indication of disorder in GO [23]. For sample C and D it is observed that the $2 D$ peak is sharper compared to sample $A$ and $B$. The intense and narrow $2 D$ peak of sample $\mathrm{D}$ indicates that the number of defects is less compared to the other samples. The FWHM of $2 D$ peak for sample A, B, C and D are $360 \mathrm{~cm}^{-1}, 155 \mathrm{~cm}^{-1}, 152 \mathrm{~cm}^{-1}$ and $59 \mathrm{~cm}^{-1}$, respectively. The reduced FWHM of 2D peak of sample D suggests the quality of reduction i.e. reduced structure with less amount of defects. The high $I 2 D / I G$ ratio can be used to predict the charge carrier mobility [24]. This $I 2 D / I G$ ratio for sample A, B, C and D is found to be $0.133,0.121,0.251$, and 0.171 , respectively. From this data, we can predict that sample C and D can show better electrical property.

The $D+G$ peak around $2890 \mathrm{~cm}^{-1}$ is a combination band and depends on the defect concentration $[10,25]$. In our case peak position of the $D+G$ band lies in the range of $2880 \mathrm{~cm}^{-1}$ to $2925 \mathrm{~cm}^{-1}$. Scientists also reported that $I 2 D / I D+G$ ratio increases due to the restoration of $s p^{2}$ hybridized structure [16]. This ratio for sample A, B, C, and D is 2.6, $4.5,5.5$, and 5 , respectively. The higher value of $I 2 D / I D+G$ indicates that there is a possibility for high restoration of $s p^{2}$ clusters in sample $\mathrm{C}$ and $\mathrm{D}$. Therefore, the large value of $I 2 D / I G$ and $I 2 D / I D+G$ is also responsible for improved electrical transport properties.

\section{$\mathrm{X}$-ray diffraction}

Figure 2 represents the XRD pattern of the RGO thin film samples (A, B, C, and D) deposited onto $\mathrm{SiO} 2(300 \mathrm{~nm}) / \mathrm{Si}$. The curve fittings for the XRD patterns of the RGO samples were done with the help of Gaussian-Lorentzian fitting profile using Origin Pro 8.5.1 software. The corresponding $2 \theta$ value for GO in the RGO samples varies from 9.770 to 12.380 which is in accordance to the previous results for GO synthesized by chemical route $[26,27]$. Reports have shown that GO has the largest interlayer distance ( $5 \AA$ - $9 \AA$ ) because of the presence of intercalated water molecules and functional groups such as hydroxyl, epoxy, and carboxyl [28].The interlayer distance for GO has been calculated, using Bragg's relation which varies from 7.1-9 $\AA$ [29]. As the films were deposited at relatively higher temperature $\left(700^{\circ} \mathrm{C}\right)$, we can see the peaks of RGO, corresponding $2 \theta$ value ranging from 15.780 to 19.370 and interlayer distance varying from 5.58-4.57 $\AA$. The shift of the $2 \theta$ peak from GO (7.1-9 $\AA$ ) to RGO (5.58-4.57 $\AA$ ) suggests that the $\mathrm{GO}$ is reduced. The shift of the GO peak towards the right is mainly dependent on the vaporization of the water molecules present between the stable GO layers which results in decrease of the interlayer distance [30]. The crystallite size for the RGO films were calculated using the Debye-Scherer formula which is shown by eqn. (1) [31]. 

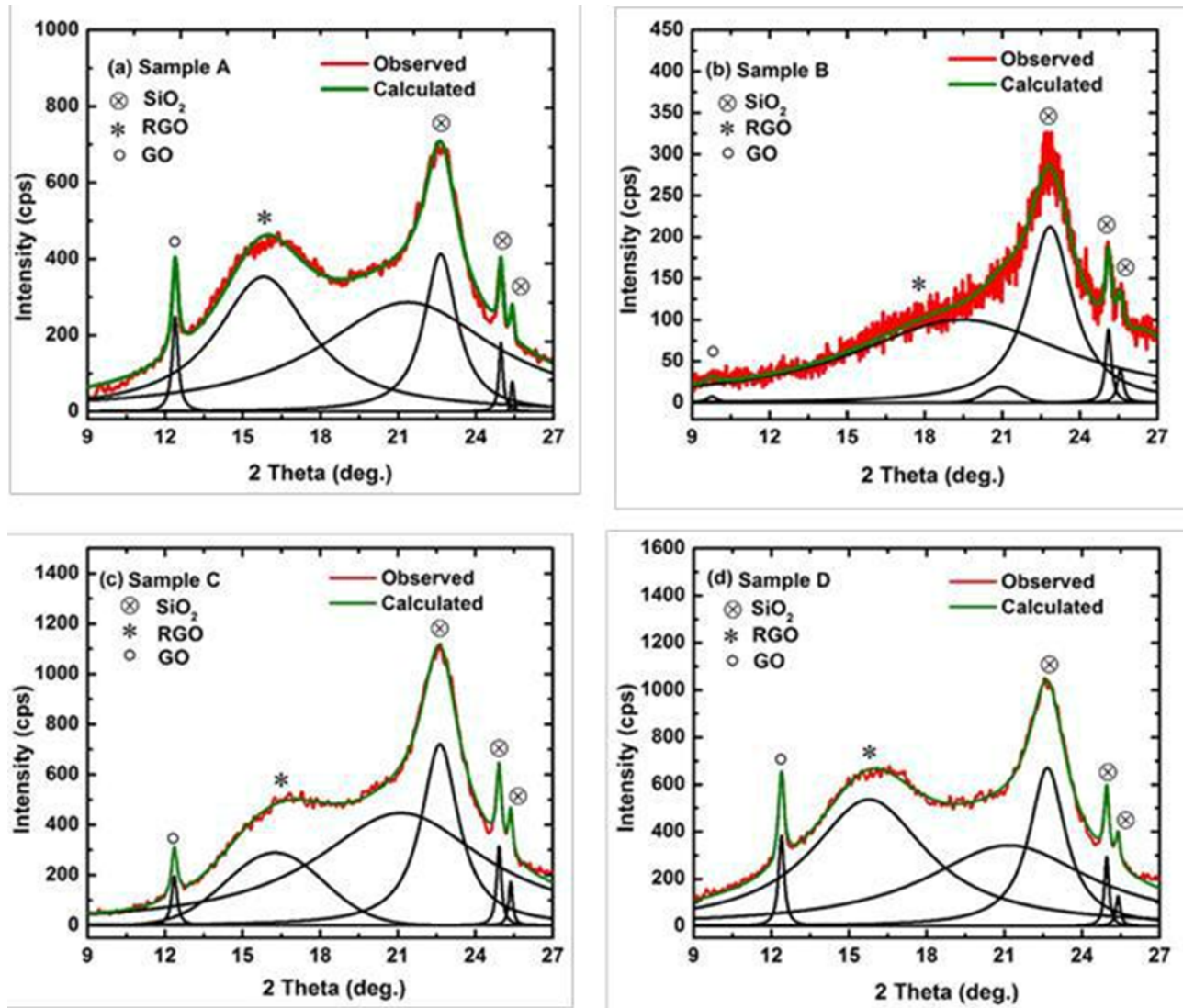

Figure 2: XRD patterns of the (a) sample $A$, (b) sample $B$, (c) sample $C$, and (d) sample $D$, interpreting interplanar separation and confirming the presence of multiphase structures of $\mathrm{GO}$ and RGO.

$$
D_{(002)}=\frac{K \lambda}{B \cos \theta}
$$

Where ${ }_{(002)}$ represents the crystallite size, $K(0.91)$ is a constant dependent on the crystallite shape, $\lambda$ is the $\mathrm{X}$-ray wavelength (1.5406 $\AA), B$ is the corrected FWHM, and $\theta$ is the scattering angle. The corrected FWHM for the corresponding GO and RGO peaks has been calculated by utilizing a strain correction, subtracting the strain from the substrate used [32].

The strain correction is represented by the eqn. (2),

$$
B=\sqrt{\left|B_{S}^{2}-B_{S u}^{2}\right|}
$$

Where, $B_{S}$ is the FWHM of the corresponding peak sample, $B_{S u}$ is the FWHM of the corresponding substrate peak.

For sample $\mathrm{A}$ and $\mathrm{C}$ there is no appreciable difference in the calculated crystallite size for RGO peaks. But for samples B and D we could see an appreciable difference in the crystallite size (8.25-15.5 $\AA$ ). The change in the crystallite size explains about the growth of the RGO thin films. With the help of Debye-Scherer equation, researchers have calculated the number of layers present in $\mathrm{GO}\left(N_{G O}\right)$ using the following eqn. (3), [33]

$$
N_{G O}=\frac{D_{(002)}}{d_{(002)}}
$$

Where $d_{(002)}$ is the interlayer distance of RGO thin films. The least number of layers (2) is found to be in sample B which is mainly dependent on the number of shots given. From the XRD pattern of sample B a broader RGO peak is visible resulting from a smaller crystallite size ( $8.25 \AA$ ) which refers the amorphous nature of the sample $\mathrm{B}$ when compared with the remaining samples (A, C and D). Detailed structural parameters of GO and RGO of four samples obtained from XRD data are given in Table 1.

\section{$\mathrm{X}$-ray photoelectron spectroscopy}

Figure 3 depicts C1s XPS data of the PLD grown RGO samples A, $\mathrm{B}, \mathrm{C}$, and $\mathrm{D}$ fabricated under different growth conditions. The curve fitting of the C1s spectra was performed using a Gaussian-Lorentzian peak profile after performing a Shirley background correction using Origin Pro 8.5.1 data plotting and acquisition software. The binding energy of the $\mathrm{C}-\mathrm{C}$ and $\mathrm{C}-\mathrm{H}$ bonding are assigned at $284.5-285 \mathrm{eV}$ and chemical shifts to $288.0 \mathrm{eV}, 286.7 \mathrm{eV}$ are typically assigned for the $\mathrm{C}=\mathrm{O}$, and -O-CH3 functional groups, respectively [34]. A peak at $283.6 \mathrm{eV}$ after deconvolution of $\mathrm{C} 1 \mathrm{~s}$ spectra for the sample $\mathrm{D}$ originates, which represents the $s p^{2}$ binding energy peak of C-C molecular bond [34]. Recent research reports indicate that graphite oxides have an epoxide group (C-O-C), which has $\mathrm{C} 1$ s binding energy similar to that of $\mathrm{C}-\mathrm{OH}$ [34]. It is also possible that there might occur a larger chemical energy shift in the $\mathrm{C}-\mathrm{O}-\mathrm{C}$ emission into the binding energy range of the $\mathrm{C}=\mathrm{O}$ emission, which can be the cause of disappearance of the epoxide group peak. The C1s spectra in Figure 3a and 3c predominantly have peaks of $s p^{3} \mathrm{C}-\mathrm{C}$ atom, $\mathrm{C}-\mathrm{H}, \mathrm{C}=\mathrm{O}$, while sample $\mathrm{B}$ has an extra - $\mathrm{O}-\mathrm{CH} 3$ instead of the $\mathrm{C}=\mathrm{O}$ ketone peak. The sample $\mathrm{D}$ has broader C1s XPS peaks with an additional peak at $283.6 \mathrm{eV}$ corresponding to the $\pi$ bonds activation energy in the graphitic network, which generally has $\sim 1 \mathrm{eV}$ less binding energy than the $\sigma$ bond. An attempt was made to fit the 
Citation: Bhaumik A, Haque A, Taufique MFN, Karnati P, Patel R, et al. (2017) Reduced Graphene Oxide Thin Films with Very Large Charge Carrier Mobility Using Pulsed Laser Deposition. J Material Sci Eng 6: 364. doi: 10.4172/2169-0022.1000364

Page 5 of 11

\begin{tabular}{|c|c|c|c|c|c|c|c|c|}
\hline & \multicolumn{2}{|c|}{ Sample A } & \multicolumn{2}{|c|}{ Sample B } & \multicolumn{2}{|c|}{ Sample C } & \multicolumn{2}{|c|}{ Sample D } \\
\hline & RGO & GO & RGO & GO & RGO & GO & RGO & GO \\
\hline Peak Position (deg) & 15.86 & 12.38 & 19.37 & 9.77 & 16.22 & 12.36 & 15.78 & 12.38 \\
\hline$d(\AA)$ & 5.587 & 7.143 & 4.57 & 9.045 & 5.45 & 7.15 & 5.61 & 7.14 \\
\hline FWHM(deg) & 4.182 & 1.51 & 9.87 & 2.03 & 4.183 & 1.77 & 5.23 & 1.67 \\
\hline Crystallite size $(\AA)$ & 19.4 & 53.5 & 8.25 & 39.71 & 19.39 & 45.64 & 15.5 & 48.37 \\
\hline Number of layers & 3.47 & 7.48 & 1.8 & 4.39 & 3.55 & 6.37 & 2.76 & 6.77 \\
\hline
\end{tabular}

Table 1: Structural parameters of $G O$ and RGO samples obtained from XRD data.
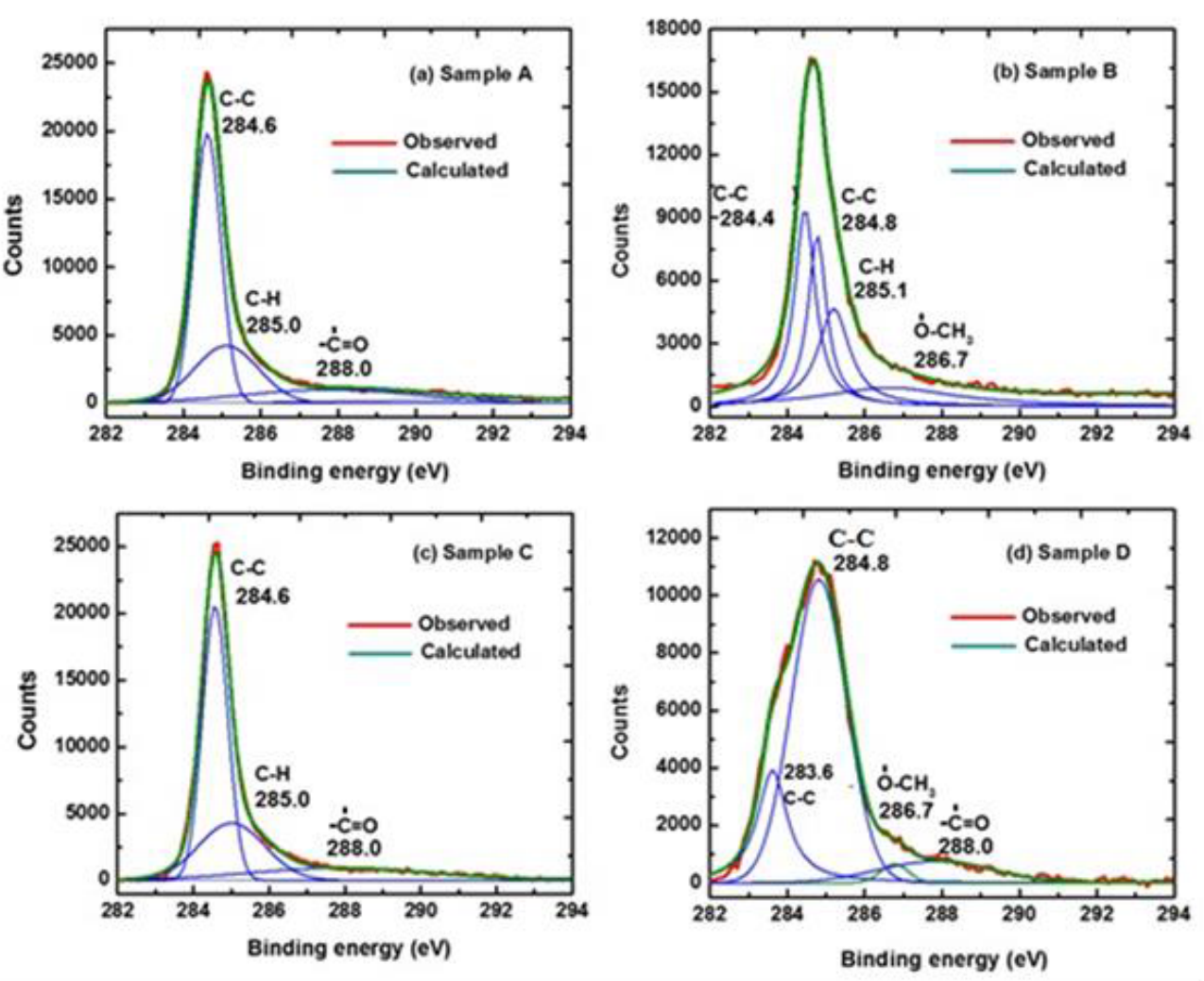

Figure 3: Deconvolution of the C1's peaks of XPS spectra of (a) sample A, (b) sample B, (c) sample C, and (d) sample D confirms the presence of C-C, $\mathrm{C}-\mathrm{H},=\mathrm{C}=\mathrm{O},-\mathrm{CH}_{3}$, and $-\mathrm{O}-\mathrm{CH}_{3}$ functional groups. From the fitted data the calculated percentage of $s p^{2} / s p^{3}$ is $54 \%, 57 \%, 54 \%$, and $87 \%$ for sample A, $\mathrm{B}, \mathrm{C}$, and $\mathrm{D}$, respectively.

expected peaks for graphene oxide at $286.6,287.6$, and $289.1 \mathrm{eV}$ but the mean square error for such a fit was much larger. The graphitic network formed by PLD technique was thereby fitted for the best goodness of fit and the peak positions and FWHM area were thus extracted. Higher values of FWHM and a broader tail towards higher binding energy is an indicative of contribution from a variety of carbon bonding configurations in the thin films. The similarity of the $\mathrm{C} 1 \mathrm{~s}$ peaks profile and position in sample $\mathrm{A}$ and $\mathrm{C}$ suggest no considerable change in the surface as well as in the depth of the sample with increasing the number of shots as the photoelectron kinetic energies of $\mathrm{C} 1 \mathrm{~s}$ is larger than $\mathrm{O} 1 \mathrm{~s}$, and so the sampling depth is larger. This proves that relatively thicker thin films of RGO grown by the PLD technique are having quite similar surface properties. All the RGO samples show a marked reduction in the intensities of all of the related oxygen peaks were indicating that the delocalized $\pi$ conjugation was restored in the samples.

The $\mathrm{C} / \mathrm{O}$ ratio was calculated for all the samples. Samples A, B, C, and $\mathrm{D}$ show a $\mathrm{C} / \mathrm{O}$ ratio of $7.2,6.25,4.28$, and 5.95 , respectively. This ratio is an indicative of the reduction in the graphene oxide structure. The above-mentioned values are appreciably lower than the reported values in a recent article, [35] suggesting that more reduction should be undertaken for complete formation of RGO. However, it should be pointed out that during thermal reduction extreme high pressure is created in the stacked layers, thereby occurring disruption of the graphitic network by loss of carbon. A pressure of $40 \mathrm{MPa}$ is generated at $300 \mathrm{C}$ as predicted by state equations, while higher pressures (130 $\mathrm{MPa})$ are generated at elevated temperatures $\left(1000^{\circ} \mathrm{C}\right)$ The evaluation of the Hamaker constant indicate that $2.5 \mathrm{MPa}$ pressure is needed to delaminate layers of stacked graphite oxide platelets [34]. An immense amount of structural defects are created during this pressure induced thermal exfoliation which also leads to the removal of carbon dioxide from graphene oxide structures [36]. Theoretical and experimental calculations have predicted a $30 \%$ loss of mass of GO during this process. This process leaves behind vacancies and various others topological defects (Stone Wales defect) throughout the basal plane in RGO [34]. The presence of these defects drastically affect the optoelectronic properties of RGO thin films by reducing the ballistic path lengths and introducing electronic charge carrier scattering moieties.

It will be discussed in electrical measurements section that the 
sample $\mathrm{D}$ is having high Hall mobility than the rest of the PLDprepared RGO samples. Though the thermal reduction is less in the sample $\mathrm{B}$, but due to the increased defect density there occurs more scattering centers for the charge carriers thereby reducing the charge carrier mobility. Carbon-oxygen signatures were detected in the XPS spectrum of the samples, but these are far diminished in the case of sample B and sample $\mathrm{D}$ than sample $\mathrm{A}$ or sample $\mathrm{C}$, and were also dwarfed by the $\mathrm{C}-\mathrm{C}$ and $\mathrm{C}=\mathrm{C}$ signals. The absence of $\mathrm{C}=\mathrm{O}$ in the sample $\mathrm{B}$ indicates that the number of pulsed laser shots is a crucial parameter for fabricating functionalized graphene oxide. With the increase in the number of shots from 100 (in sample B) to 500 (in sample D) evolution of $\mathrm{C}=\mathrm{O}$ peak occurs. Further increase in the number of PLD shots completely replaces the $-\mathrm{O}-\mathrm{CH} 3$ to $\mathrm{C}=\mathrm{O}$ functional group. The amount of $s p^{2}$ content is generally indicated by the $\mathrm{C}-\mathrm{C}$ peak, while the oxygen-containing functional groups give rise to $s p^{3}$ hybridized states [5]. The presence of $s p^{3}$ defect sites cause a distortion in the $\pi$ continuum cloud of the intrinsic $\pi$ electronic states of the $s p^{2}$ sites. Therefore the $s p^{2}$ fraction in RGO thin films provide a valuable insight to its optoelectronic properties and is commonly referred to as the reduction efficiency [5]. The $s p^{2}$ fraction is calculated from the XPS high resolution data. This is done by considering the ratio of the integrated area of the peak corresponding to the $\mathrm{C}$ - $\mathrm{C}$ bond to the total area under the C1s XPS spectra. The percentage of the carbon $s p^{2}$ fraction is determined by the following equation [35]:

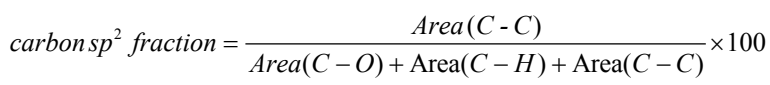

The percentage of $s p^{2}$ fraction calculated from XPS is $54 \%, 57 \%$, $54 \%$, and $87 \%$ for sample A, B, C, and D, respectively. Increased $s p^{2}$ fraction in sample $\mathrm{D}$ is an indicative of the restoration of the $\pi$ network in the RGO structure, which will be the determining factor for increased electrical mobility observations.

\section{Electrical transport properties}

Figure $4 \mathrm{a}$ represents the resistance- temperature (R-T) characteristics of the RGO thin film samples. Electrical measurements indicate good electronic properties of the as-synthesized RGO thin films. Within the voltage range of -100 to $100 \mathrm{mV}$, the $I-V$ curves are Ohmic which allows to get more reliable resistance measurements of the samples at different temperatures. Four probe measurement setup also helps avoid the contact resistance and lead resistance. To better understand the electronic properties R-T data have been collected over a broad range of temperature ( $5 \mathrm{~K}$ to $400 \mathrm{~K}$ ). In all the samples the value of resistance increases nonlinearly with decrease in temperature which implies the semiconducting nature of the thin films. With decreasing temperature from $400 \mathrm{~K}$ to $5 \mathrm{~K}$, we have noticed two completely different types of rate of change of resistance i.e. at higher temperature region the resistance increases slowly and at lower temperature region it increases rapidly. So the transport mechanism is thought to be following two different kind of models. Researchers have experimentally found that at high and low-temperature regimes mode of electrical transport mechanism is dominated by band gap and variable range hopping processes, respectively [37]. Resistance vs. temperature data of all the samples in temperature range $5 \mathrm{~K}$ to $190 \mathrm{~K}$ elucidate that the transport mechanism is dominated by Efros-Shklovskii variable range hopping (ES VRH) and from range $190 \mathrm{~K}$ to $400 \mathrm{~K}$ the data best fit with Arrhenius equation. As at lower temperature activated type of conduction is not possible in the RGO thin films, the variable range hopping mechanism plays a significant role in the conduction process. The $s p^{3}$ hybridization of $\mathrm{C}$ atoms and discontinuous $s p^{2}$ clusters in $s p^{3}$
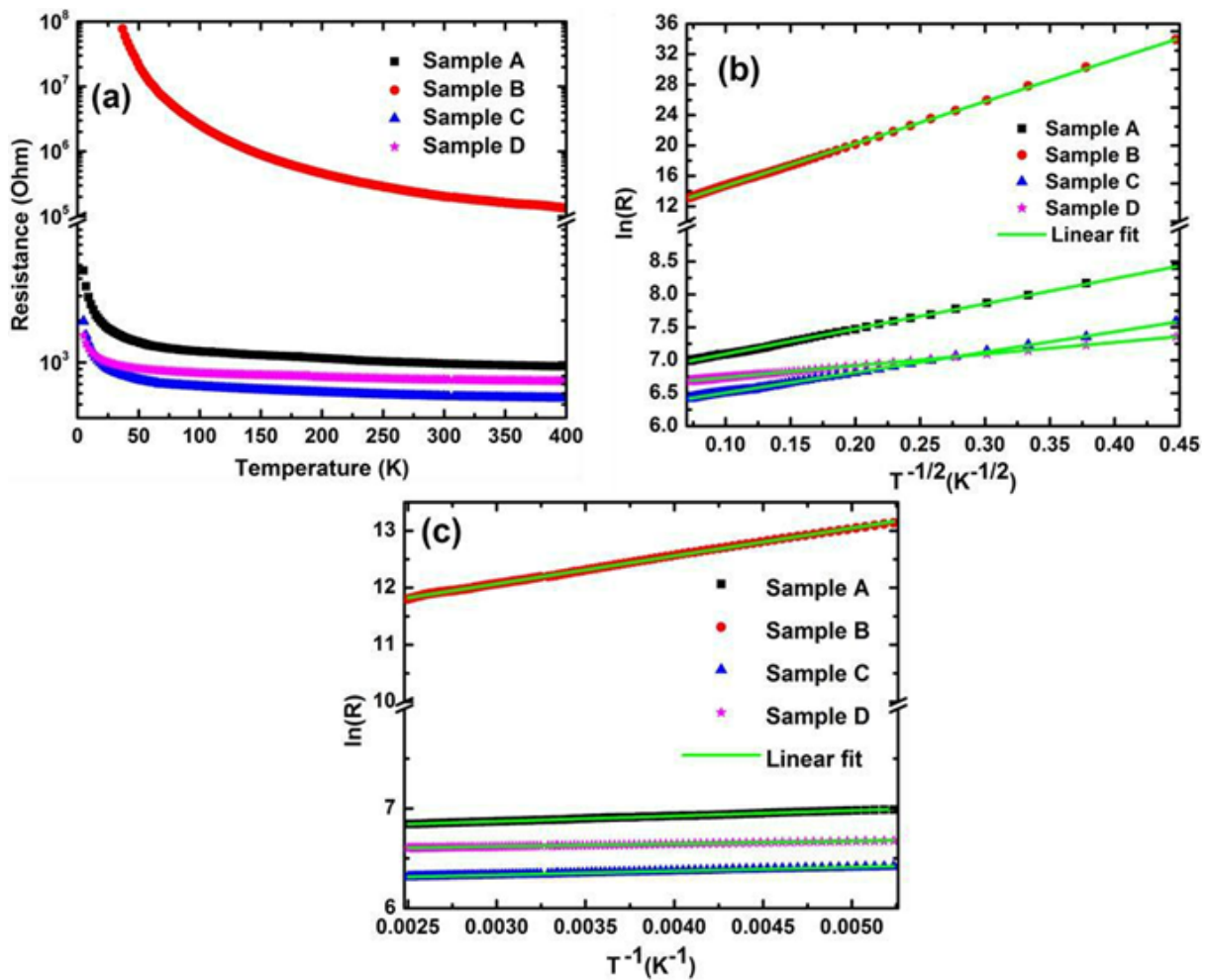

Figure 4: (a) Temperature ( $T$ ) dependence of electrical resistance of samples $A, B, C$, and $D$ in the range $5 \mathrm{~K}<\mathrm{T}<400 \mathrm{~K}$. (b) ES VRH region is identified by plotting $\ln (\mathrm{R})$ as a function of $T^{-1 / 2}$ in the temperature range $5 \mathrm{~K}<\mathrm{T}<190 \mathrm{~K},(\mathrm{c}) \ln (\mathrm{R})$ as a function of $T^{-1}$ in the temperature range $190 \mathrm{~K}<\mathrm{T}<400 \mathrm{~K}$ to illustrate the band-gap dominated Arrhenius-like temperature dependence transport mechanism. 
matrix play a major role for the insulating property of GO. Scientists suggested that new domains of $s p^{2}$ clusters are formed by the removal of oxygen groups due to the reduction of GO. This gives the percolation path between domains already present. Initial $s p^{2}$ clusters of the GO do not take part in conduction [38].

The new $s p^{2}$ clusters, which were formed by the subsequent reduction reaction of the thin film at high temperature $\left(700^{\circ} \mathrm{C}\right)$, help in electron transport process. Newly formed $s p^{2}$ clusters can be considered electronically as isolated states that aid in hopping of the free electrons. As the number of $s p^{2}$ clusters is finite so the conductance in the as grown RGO film is measurable. To determine the conduction mechanism in the RGO samples we fitted our results with various conductivity mechanism models. At low temperature regime we found that the data best fit to a straight line with ES-VRH model, shown in Figure $4 \mathrm{~b}$, compared to Mott-VRH or 3D models [39]. ES model was considered from the specific form of single particle density of states in Coulomb gap to include the effects of Coulomb interactions and modified Mott's argument of variable range hopping [40]. From this model a relationship between the conductivity and temperature is formed. This relationship can be expressed in terms of resistance, $R$ and temperature, $T$ by eqn. (5) [5].

$$
R=R_{0} e^{\left(\frac{T_{0}}{T}\right)^{1 / 2}}
$$

Where $T_{0}$ is the characteristic temperature and the exponent $1 / 2$ is independent of dimensionality of the sample. This characteristics temperature $T_{0}$ can be used to find out the localization length, $\xi$ using eqn. (6) [41].

$$
T_{0}=\frac{\beta e^{2}}{4 \pi \varepsilon \varepsilon_{0} k_{B} \xi}
$$

Where $\beta=2.8$ is a constant, $k_{B}$ is the Boltzmann constant, $e$ is the charge of an electron, ${ }_{0}$ is the vacuum permittivity, and $\varepsilon$ is the effective dielectric constant of RGO thin films on $\mathrm{SiO}_{2}$. From different studies we can estimate the value of $\varepsilon$ to be 3.5 for our RGO samples $[28,42,43]$. From the fitted data demonstrated in Figure $4 \mathrm{~b}$ we obtained characteristic temperature which allows to calculate the localization lengths of corresponding samples. From the values of localization length reported in Table 2 we can infer that the longest localization length has been found in the sample with larger conductivity i.e. sample D. Minimum localization length is obtained in the least conductive sample i.e. sample B.

Room temperature Hall mobility measurement was performed in all the samples. Only sample D has shown a considerable amount $(372$ $\mathrm{cm}^{2} \mathrm{~V}^{-1} \mathrm{~s}^{-1}$ ) and sample $\mathrm{C}$ has shown a meager amount of Hall mobility. The Hall mobility of sample D calculated from the Magnetic field vs Hall voltage data collected from four probe measurement setup. It has been experimentally shown that the fraction of $s p^{2}$ sites in RGO increases with increasing localization length [42]. According to Raman analysis sample D has less defect states and as it is having highest $s p^{2}$ fraction among all the samples, noticeable mobility was observed in this sample. Another possible explanation for improved mobility could

\begin{tabular}{|c|c|c|c|}
\hline Sample Name & $\begin{array}{c}\text { Localization Length } \\
(\mathbf{n m})\end{array}$ & $\begin{array}{c}\text { Activation Energy } \\
(\mathbf{m e V})\end{array}$ & $\begin{array}{c}\text { Hopping Energy } \\
(\mathbf{m e V})\end{array}$ \\
\hline Sample A & 916 & 4.7 & 4.2 \\
\hline Sample B & 4 & 42.1 & 17.7 \\
\hline Sample C & 1401 & 3.4 & 3.9 \\
\hline Sample D & 4227 & 2.3 & 3.7 \\
\hline
\end{tabular}

Table 2: Electrical parameters of RGO thin film samples A, B, C, and D. be a longer localization length which is the outcome of the shortened barrier heights for electron transportation among the $s p^{2}$ clusters. Hopping energy $(E h)$ is an important parameter in ES VRH model of $2 \mathrm{D}$ materials. The hopping energies for all the samples are calculated by using the following formula represented by eqn. (7) and given in Table 2 [30].

$$
\sigma \sqrt{T}=A e^{-\frac{E_{h}}{k_{B} T}}
$$

Where $A$ is a constant, and $\sigma$ is the conductivity. As lower hopping energy always facilitates the transportation of electrons in the sample, we found the maximum conductivity in sample D where minimum hopping energy is obtained. On the other hand, as sample $\mathrm{B}$ demonstrates maximum hopping energy, maximum resistivity is observed in this sample.

At higher temperature regime $(190-400 \mathrm{~K})$ the data is analyzed based on the relationship between temperature and resistance which is represented in eqn. (8) [17].

$$
R=R_{0} e^{\frac{E_{g}}{k_{B} T}}
$$

Where $E g$ is the activation energy. The plotted data of natural logarithm of resistance $\ln (R)$ versus inverse of temperature $T^{-1}$ best fit to a straight line in Figure $4 \mathrm{c}$. This implies that at higher temperature regime band-gap dominated Arrhenius-like temperature dependence plays the major role in transport mechanism. Using the slope of the fitted lines the calculated 15 activation energy is found to be in a range from $2.35 \mathrm{meV}$ to $37.54 \mathrm{meV}$ which is comparable with the values reported previously $[44,45]$.

The activation energy is minimum in case of sample $\mathrm{D}$. So, at room temperature it is easier for the electrons in the donor atoms lying in the crystal to reach to the conduction band. Completely opposite phenomenon occurs in the case of sample B with higher activation energy and this scenario is portrayed in Figure 5. The work of Pearson and Bardeen also showed a decrease in activation energy which results an increase in carrier concentration [46]. For each kind of defect/ impurity, there should be a specific characteristic ionization energy also termed as activation energy. This energy is fixed under normal operational conditions [47]. So, the value of the activation energy is dependent only on the nature of the intrinsic and extrinsic defects/ impurities in the samples. The variance in the activation energy of
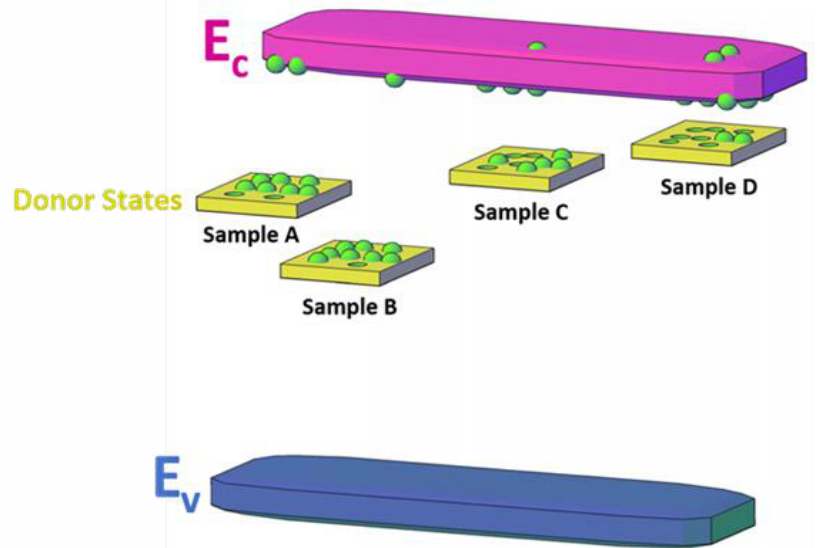

Figure 5: Depicts the activation energy of the samples synthesized by PLD technique. 
our samples implies that the nature and the level of the defects and impurities are dependent on different growth parameters.

The decrease in carbon $s p^{2}$ fraction leads to an increase in $R$. Among all the samples maximum value of $R$ is found in sample $B\left(\sim 10^{8}\right.$ $\Omega)$ while the minimum is found in sample $\mathrm{A}\left(\sim 10^{3} \Omega\right)$ demonstrating that the value of $R$ can be tuned by more than 5 orders of magnitude by varying the carbon $s p^{2}$ fraction which supports the information illustrated from Raman spectroscopy. The decrease in resistance with increasing $s p^{2}$ fraction demonstrates that the restoration of $\pi-\pi$ bond improves charge percolation pathways in the RGO sheet. It has also been observed that such improvement occurs at the expense of increasing topological defects in the samples [48,49]. The salient features of improved mobility can be accounted for (i) less hopping energy, (ii) longer localization length, (iii) minimal defect density, and (iv) smaller activation energy. Better conductivity of our samples suggests the formation of numerous $s p^{2}$ clusters by the removal of oxygen groups in the $s p^{3}$ matrix [38]. The presence of the huge number of small $s p^{2}$ clusters can also be proven by the blue shifting of widened PL peak.

\section{Photoluminescence spectroscopy}

Figure 6 represents the PL spectra of the RGO samples A, B, C, and D synthesized by PLD technique. We have represented the PL after subtracting from the background PL signal of the substrate measured under identical conditions. RGO is a graphene sheet functionalized with oxygen groups on the basal plane and at the edges, thereby exhibiting interesting steady-state PL properties. The PL spectra show a broad PL region between $1.25 \mathrm{eV}$ to $3.00 \mathrm{eV}(410 \mathrm{~nm}$ to $990 \mathrm{~nm})$ and is also reported to be a characteristic spectrum for oxygen plasmatreated, mechanically exfoliated, single-layer graphene sheet [38]. The prominent PL peak seen in sample D is observed at $2.3 \mathrm{eV}$ which moves towards lower energy values for all other synthesized samples. Increased $s p^{2}$ fraction (as calculated by XPS) in sample D decreases the $\mathrm{PL}$ intensity and exhibits blue fluorescence (as the peaks move towards right). The increased PL intensity in sample $\mathrm{C}$ is due to less reduction (C/O ratio) as observed by XPS studies. The sharper peak profile for the $2 D$ peak also influences the PL spectra. A decrease in FWHM of the $2 D$ peak also corresponds to blue fluorescence in the PL spectra.

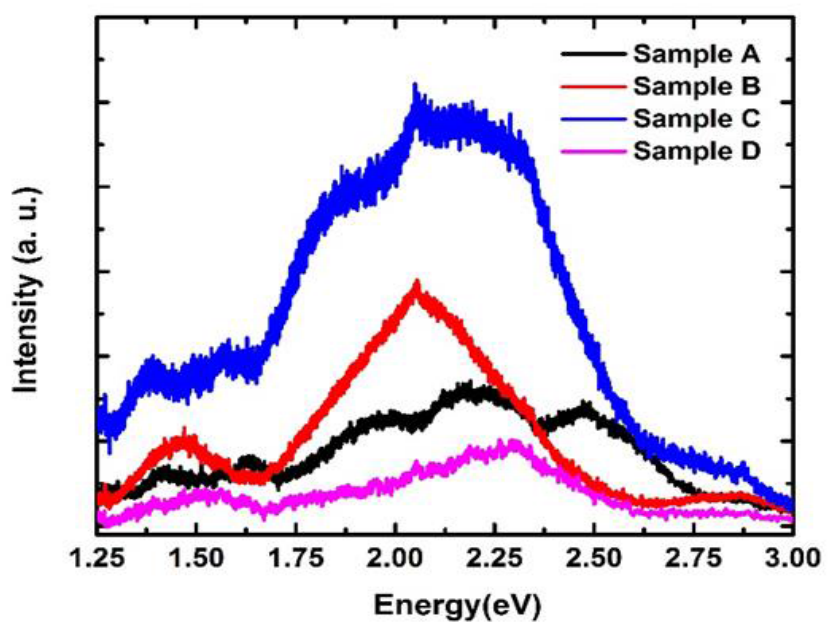

Figure 6: $\mathrm{PL}$ spectra of all the RGO samples after subtracting from the background signal of the substrate. The reduced intensity and the higher energy position of the PL peak of sample $D$ imply relatively high $\mathrm{C} / \mathrm{O}$ ratio and high $s p^{2}$ hybridized carbon fraction compared to the other samples.
This can be due to the reason that a sharper $2 D$ peak is an indicative of reduced defect regions in the layered structure of GO. First-principle calculation suggests a large fraction of the $\mathrm{C}$ atoms in the hydroxyl and epoxy units are bonded to each other to form strips of $s p^{2}$ carbon atoms in GO compounds [46]. In GO compounds, containing a mixture of $s p^{2}$ and $s p^{3}$ moieties, the optoelectronic properties are mainly determined by the $\pi$ and $\pi^{*}$ electronic states of the $s p^{2}$ sites, which lie within the $\sigma-\sigma^{*}$ gap [38]. The $\pi$ bonding is weaker and has a lower formation energy than the $\sigma$ bond, Therefore, there occurs the presence of a large density of disorder-induced localized states in the two-dimensional structure of as synthesized GO and RGO. There are electronic states associated with the functionalized carbon atoms situated in the basal plane and edges of the structure which gives rise to interesting PL features. It is well studied that the interactions of the $\pi$ states are strongly dependent on their projected dihedral angles. There also occurs the formation of these structurally disordered localized electronic states in the band tail or deep states in the $\pi-\pi^{*}$ electronic gap [38]. The optical transitions resulting from these states result in a broader absorption or emission band and is evident from the PL spectra. The deoxygenation process which leads to reduction (formation of RGO) decreases the density of these disorder-induced localized states and forms well defined $s p^{2}$ clusters. This causes a decrease in the intensity of the PL emission with reduction. The creation of RGO gives rise to formation of numerous smaller sized $s p^{2}$ clusters which plays an important role in the variable hopping range electronic conduction. The formation of new $s p^{2}$ clusters with reduction also broadens and reduces the intensity of PL spectra. The formation of these miniature sized $s p^{2}$ clusters in RGO provides percolation pathways between $s p^{2}$ clusters already present. Thus, it can be realized that the reduction of GO to RGO gives rise to zeroband gap regions. There are still some oxygen functional groups which remains unreduced during this process, which acts as scattering sites. The ratio of the zero gap $s p^{2}$ clusters to the $s p^{3}$ clusters in the RGO is quite high which results in the quenching of the PL signal due to the phenomena of weak carrier confinement [38]. The original GO consists of numerous disorder induced defect states within the $\pi-\pi^{*}$ gap and exhibits a broad prominent PL spectrum centered at 500-600 nm. After deoxygenation, the number of disorder-induced states within the $\pi-\pi^{*}$ gap decreases, and an increased number of cluster-like states from the newly formed small and isolated $s p^{2}$ domains are formed. The electronhole recombination among these $s p^{2}$ cluster-like states exhibits blue fluorescence at shorter wavelengths with a narrower bandwidth. The heterogeneous electronic structures of GO and RGO with variable $s p^{2}$ and $s p^{3}$ hybridizations through reduction gives rise to broader and interesting featured PL spectra in the PLD synthesized samples.

\section{Discussion}

The typical broadening of $D$ peaks and then their gradually overlapping with $G$ peaks in the RGO thin films indicate a successful hybridization phenomena. Varying the number of pulsed laser shots altered the physical properties of the fabricated RGO films. Kinetic energy of the atoms, molecules or ions ablated form the target are mainly dependent on the laser parameters and target characteristics (graphite target). Graphitic entities were being formed onto the $\mathrm{SiO} 2$ $(300 \mathrm{~nm}) / \mathrm{Si}$ substrates after laser ablation of graphite target. When the plume comes in contact to another surface (substrate), it condenses to form thin film which mainly depends on the surface energy of the substrate. Depending on the thermodynamics and surface energies between the film and substrate and also film-interface energy it follows three different growth mechanisms [50]. Increased surface energy of the substrate facilitated random movement of $s p^{2}$ clusters on the substrate thereby forming layered GO. GO sheets could be thought as graphene 
that was decorated with oxygen-containing functional groups, these functional groups could be gradually removed and $s p^{2}$ conjugated graphene network would partially be restored during the thermal reduction process, which would result in the transition from insulator to semimetal in RGO [51]. Recently, we have also reported formation of graphene, RGO, and GO using pulsed laser annealing technique [51]. This transition would change the manners of charge transportation and generate tunable electronic properties within the thin films as presented in this research article. Calculation of inter planar distances from XRD plot confirms the presence of multi-phase structures of GO and RGO in the thin films. The broadened XRD peak in the case of sample B indicates the formation of amorphous $s p^{2}$ clusters, thereby having an enormous resistance. Raman spectroscopy was carefully analyzed to extract interesting structural properties in the samples pertaining to defect states. The sharper $2 D$ peak profile was an indicative of better electrical mobility in the sample D. A layer of graphene is introduced to enhance the charge carrier mobility of the PLD deposited RGO layer. Pulsed laser deposition (unlike CVD) is a non-equilibrium processing technique, where the energies of the ablated species are $\sim 100 \mathrm{kT}$. Increasing the thickness of the deposited layer will introduce defects (Stone-Wales and functionalization defects) in the structure of the RGO layer which will impede the charge carrier mobility. So, the thickness of the deposited thin film is an important factor governing the electronic properties of RGO. In this report, the highest value of Hall mobility was achieved in the 500 pulsed laser shots sample (sample D). Detailed experiment of thickness dependent mobility in RGO thin films is under investigation and will be reported elsewhere. Again, the calculated defect densities indicate an increase with increasing number of PLD shots. FWHM of the $2 D$ peak is an indicative of the degree of disorderness in the graphene (or GO and RGO structures). This peak is the characteristic of the double resonance transition arising from the generation of two phonons with wave vectors $+k$ and $-k$ (travelling in opposite direction). The FWHM of $2 D$ peak for sample A (5000 shots), B (100 shots), C (10,000 shots) and D (500 shots on graphene) are $360 \mathrm{~cm}^{-1}, 155 \mathrm{~cm}^{-1}, 152 \mathrm{~cm}^{-1}$ and $59 \mathrm{~cm}^{-1}$, respectively. This clearly indicates that the sample A has the highest degree of disorderness as compared to the other synthesized RGO thin films. The partial pressure of oxygen gas maintained throughout the pulsed laser deposition time was $10^{-5}$ torr. With the increase in the number of PLD shots there is an increase in the size of the $s p^{3}$ moieties formed after functionalization of the edges and basal plane of RGO. This increases the FWHM of the $2 \mathrm{D}$ peak and also the $I D / I G$ ratio and is evident from the Raman spectroscopy of samples A and B. With the further increase in the number of the PLD shots there occurs an increased concentration of the $s p^{2}$ entities, thereby decreasing the FWHM of the $2 D$ Raman peak. This is completely in accordance with PLD surface kinematics. Though the defect density was least in sample B, the resistance was appreciably larger due to the presence of isolated $s p^{2}$ clusters. In addition to that, the measured electrical parameters such as localization length, hopping energy, and activation energy is not favorable to produce a large charge carrier mobility. In case of sample $\mathrm{D}$, the incorporation of graphene as a seed layer helps attain high $s p^{2}$ carbon fraction as well as large $I 2 D / I G$ ratio along with reduced FWHM of the $2 D$ peak of Raman spectrum. The combination of these favorable parameters contribute to the enhancement in charge carrier mobility and we observed the maximum amount of charge carrier mobility in this sample. Furthermore, an increased number of miniature $s p^{2}$ moieties favors charge carrier variable range hopping mechanism. Other important factors that played a crucial role in the exuberant increase in charge carrier mobility for sample D were: increased localization length (4227 $\mathrm{nm})$, decrease in hopping energy $(3.7 \mathrm{meV})$, and decrease in activation energy $(2.3 \mathrm{meV})$. Although the ratio of $s p^{2} / s p^{3}$ carbon fraction is less in sample $\mathrm{C}$, but due to the favorable values of localization length, hopping energy, activation energy, and $I 2 D / I G$ ratio it showed a moderate value of charge carrier mobility. In sample A though the measured values of electrical characterization parameters are close to those of sample C but the large FWHM of $2 D$ Raman peak and small value of $I 2 D / I G$ result no measureable mobility in this sample. Surface defects usually act as scattering centers as they pin the electronic charge flow. The $\mathrm{C} / \mathrm{O}$ ratio as calculated from XPS data suggests reduced surface defects due to moderate evolution of $\mathrm{CO} 2$ from the layered RGO structure. The low temperature resistance measurements suggests that the density of states (DOS) near the Fermi level $(E F)$ is variable and it disappears linearly with energy for a two dimensional (2D) system (ES-VRH) [51]. When an electron hops from one site to another leaving behind a hole, the system must have enough energy to overcome this electron-hole Coulomb interaction for reduced resistance [5]. The non-constancy of the DOS predicts presence of disorder in the layered samples, is also presumably observed in Raman spectroscopy, which in particular is sensitive to $s p^{2}$ electronic states for the green laser used. The decreased activation energy as calculated in sample D by Arrhenius equation is also an indicative of increased concentration of $s p^{2}$ clusters near the $\pi^{*}$ electronic state. Qualitatively it can also be inferred from the broader blue fluorescence in PL spectra that the sample D is having more $s p^{2 \%}$ than the rest of the PLD grown samples. A $s p^{2}$ fraction of $87 \%$ was also being calculated from XPS analysis which reinforces the above mentioned fact. Detailed structural and electronic correlation parameters found through this study are given in Table 3.

The dark gray balls in the structural drawing represents $\mathrm{C}$, the yellow ones $\mathrm{H}$, and the red ones $\mathrm{O}$ [51].

Figure 7 is a schematic representation of the structural, optical, and electrical properties of the sample D along with a snapshot of the sample grown in the research facility. The pictorial representation of PL spectra represented in this figure indicates blue florescence due to the presence of innumerous $s p^{2}$ clusters, which also favors VRH mechanism. During synthesis of RGO samples the as-deposited RGO sheets could cover grain boundaries and wrinkles uniformly. This could facilitate easy movement of charge carriers underneath the film to cross over the grain boundaries with the assistance of RGO sheet via variable-range hopping process through the highly conductive graphene islands. In addition, the thermal reduction resulted in conductivity enhancement of RGO which facilitated the hopping process of charge carrier, thus leading to increased value of charge carrier Hall mobility.

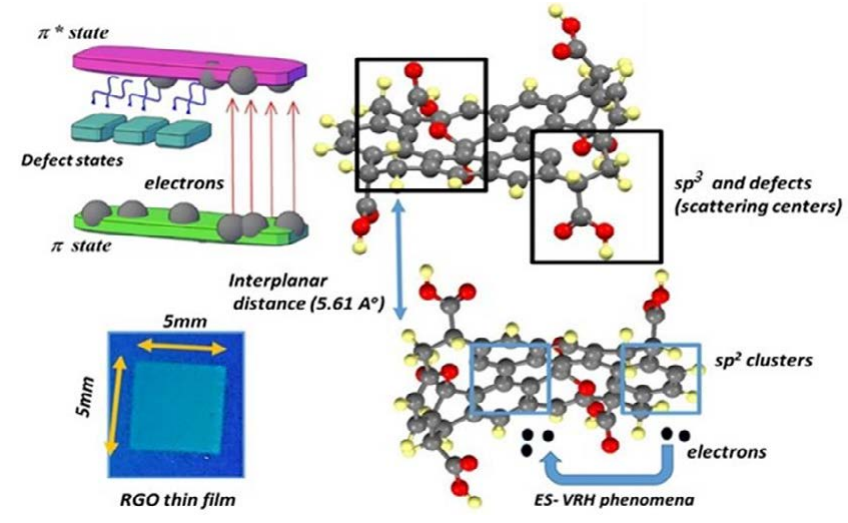

Figure 7: Schematic diagram that dictates the structural, optical, and electrical properties of the high mobility sample $D$ along with a snapshot of the sample grown in the research facility. 
Citation: Bhaumik A, Haque A, Taufique MFN, Karnati P, Patel R, et al. (2017) Reduced Graphene Oxide Thin Films with Very Large Charge Carrier Mobility Using Pulsed Laser Deposition. J Material Sci Eng 6: 364. doi: 10.4172/2169-0022.1000364

Page 10 of 11

\begin{tabular}{|c|c|c|c|c|c|c|}
\hline Sample Name & ID/IG & I2D/IG & $I 2 D / I D+G$ & FWHM of $2 D\left(\mathrm{~cm}^{-1}\right)$ & $s p^{2} / s p^{3}(\%)$ & Hall mobility $\left(\mathrm{cm}^{2} \mathrm{~V}^{-1} \mathrm{~s}^{-1}\right)$ \\
\hline Sample A & 1.65 & 0.133 & 2.6 & 360 & 54 & Small \\
\hline Sample B & 0.97 & 0.121 & 4.5 & 155 & 57 & Small \\
\hline Sample C & 1.57 & 0.251 & 5.5 & 152 & 54 & 34 \\
\hline Sample D & 1.14 & 0.171 & 5 & 59 & 87 & 372 \\
\hline
\end{tabular}

Table 3: Structural and electronic properties of samples A, B, C, and D.

\section{Conclusions}

Large area RGO thin films have been grown using PLD having a very large carrier mobility of $372 \mathrm{~cm}^{2} \mathrm{~V}^{-1} \mathrm{~s}^{-1}$. Larger carrier mobility has been observed with a larger fraction of $s p^{2}$ carbon fraction and a narrower Raman $2 D$ peak profile in PLD grown RGO thin films. The ratio of $s p^{2}$ and $s p^{3}$ hybridized carbon atoms in functionalized graphene plays a major role in controlling the electronic properties. At lower temperature the charge carriers are having limited thermal energy to move in the plane, which can be drastically reduced with the increase in defect density with reduction. Increased $s p^{2}$ fraction helps ES-VRH mechanism thereby introducing exuberant electrical mobility. The fabrication of large area RGO thin films with very large carrier mobility fabricated by PLD method and understanding the correlation with the structural and electrical properties in reduced graphene oxide thin film will be very useful for high mobility electronic devices and could open a roadmap for further extensive research in functionalized 2D materials.

\section{Acknowledgements}

The authors would like to acknowledge Dr. William Mitchel, Air Force Research Laboratory for providing CVD grown graphene. The authors 1 through 4 contributed equally to this work. The authors would also like to acknowledge the NSF Grant (DMR-08211593) supporting this research.

\section{References}

1. Novoselov KS, Geim AK, Morozov SV, Jiang D, Zhang Y, et al. (2004) Electric field effect in atomically thin carbon films. Science 306: 666-669.

2. Lee JW, Hall AS, Kim JD, Mallouk TE (2012) A facile and template-free hydrothermal synthesis of $\mathrm{Mn} 3 \mathrm{O} 4$ nanorods on graphene sheets for supercapacitor electrodes with long cycle stability. Chemistry of Materials 24: 1158-1164.

3. Li D, Kaner RB (2008) Graphene-based materials. Nat Nanotechnol 3: 101.

4. Zhu J, Zhang H, Kotov NA (2013) Thermodynamic and structural insights into nanocomposites engineering by comparing two materials assembly techniques for graphene. ACS nano 7: 4818-4829.

5. Joung D, Khondaker SI (2012) Efros-Shklovskii variable-range hopping in reduced graphene oxide sheets of varying carbon s p 2 fraction. Physical Review B 86: 235423.

6. Zhang Y, Tan YW, Stormer HL, Kim P (2005) Experimental observation of the quantum Hall effect and Berry's phase in graphene. Nature 438: 201-4.

7. Robertson J (2006) Amorphous carbon. Advances in Physics 35:317-374.

8. Wang ZF, Li Q, Zheng H, Ren H, Su H, et al. (2007) Tuning the electronic structure of graphene nanoribbons through chemical edge modification: $A$ theoretical study. Physical Review B 75: 113406.

9. Ohta T, Bostwick A, Seyller T, Horn K, Rotenberg E (2006) Controlling the electronic structure of bilayer graphene. Science 313: 951-954.

10. Stankovich S, Dikin DA, Piner RD, Kohlhaas KA, Kleinhammes A, et al. (2007) Synthesis of graphene-based nanosheets via chemical reduction of exfoliated graphite oxide. Carbon 45: 1558-1565.

11. Tello PG, Castano FJ, O'Handley RC, Allen SM, Esteve M, et al. (2002) Ni$\mathrm{Mn}-\mathrm{Ga}$ thin films produced by pulsed laser deposition. Journal of applied physics 91: 8234-8236.

12. Bonaccorso F, Bongiorno C, Fazio B, Gucciardi PG, Marago OM, et al. (2007) Pulsed laser deposition of multiwalled carbon nanotubes thin films. Applied Surface Science 254: 1260-1263.

13. Habermeier HU (1993) Pulsed laser deposition-a versatile technique only for high-temperature superconductor thin-film deposition? Applied surface science 69: 204-211.

14. Kumar I, Khare A (2014) Multi-and few-layer graphene on insulating substrate via pulsed laser deposition technique. Applied Surface Science 317: 10041009.

15. Trusovas R, Ratautas K, Račiukaitis G, Barkauskas J, Stankevičienè I, et al. (2013) Reduction of graphite oxide to graphene with laser irradiation. Carbon 52: 574-582.

16. Mathew S, Chan TK, Zhan D, Gopinadhan K, Barman AR, et al. (2011) The effect of layer number and substrate on the stability of graphene under MeV proton beam irradiation. Carbon 49: 1720-1726.

17. Ferrari AC, Robertson J (2000) Interpretation of Raman spectra of disordered and amorphous carbon. Physical review B 61: 14095.

18. Krishnamoorthy K, Veerapandian M, Yun K, Kim SJ (2013) The chemical and structural analysis of graphene oxide with different degrees of oxidation. Carbon 53: 38-49.

19. Yan J, Zhang Y, Kim P, Pinczuk A (2007) Electric field effect tuning of electronphonon coupling in graphene. Physical review letters 98: 166802.

20. Wang L, Park Y, Cui P, Bak S, Lee H, et al. (2014) Facile preparation of an n-type reduced graphene oxide field effect transistor at room temperature. Chemical Communications 50: 1224-1226.

21. Al-Jishi R, Dresselhaus G (1982) Lattice-dynamical model for graphite. Physical Review B 26: 4514-4522.

22. Gupta A, Chen G, Joshi P, Tadigadapa S, Eklund PC (2006) Raman scattering from high-frequency phonons in supported $n$-graphene layer films. Nano letters 6: 2667-2673.

23. Malard LM, Pimenta MAA, Dresselhaus G, Dresselhaus MS (2009) Raman spectroscopy in graphene. Physics Reports 473: 51-87.

24. Su CY, Xu Y, Zhang W, Zhao J, Tang X, et al. (2009) Electrical and Spectroscopic Characterizations of Ultra-Large Reduced Graphene Oxide Monolayers. Chemistry of Materials 21: 5674-5680.

25. Zhan D, Ni Z, Chen W, Sun L, Luo Z, et al. (2011) Electronic structure of graphite oxide and thermally reduced graphite oxide. Carbon 49: 1362-1366.

26. Some S, Kim Y, Yoon Y, Yoo H, Lee S, et al. (2013) High-quality reduced graphene oxide by a dual-function chemical reduction and healing process. Scientific reports 3: 1929

27. Marcano DC, Kosynkin DV, Berlin JM, Sinitskii A, Sun Z, et al. (2010) Improved synthesis of graphene oxide. ACS nano 4: 4806-4814.

28. Jung I, Vaupel M, Pelton M, Piner R, Dikin DA, et al. (2008) Characterization of thermally reduced graphene oxide by imaging ellipsometry. The Journal of Physical Chemistry C 112: 8499-8506.

29. Mikhailov S ed (2011) Physics and Applications of Graphene - Experiments InTech.

30. Cullity BD, Weymouth JW (1957) Elements of X-ray Diffraction. American Journal of Physics 25: 394-395.

31. McKeehan M, Warren BE (1953) X-Ray Study of Cold Work in Thoriated Tungsten. Journal of Applied Physics 24: 52.

32. Ju HM, Huh SH, Choi SH, Lee HL (2010) Structures of thermally and chemically reduced graphene. Materials Letters 64: 357-360.

33. Eda G, Fanchini G, Chhowalla M (2008) Large-area ultrathin films of reduced graphene oxide as a transparent and flexible electronic material. Nature nanotechnology 3: 270-274.

34. Becerril HA, Mao J, Liu Z, Stoltenberg RM, Bao Z, et al. (2008) Evaluation of solution-processed reduced graphene oxide films as transparent conductors. ACS nano 2: 463-70. 
Citation: Bhaumik A, Haque A, Taufique MFN, Karnati P, Patel R, et al. (2017) Reduced Graphene Oxide Thin Films with Very Large Charge Carrier Mobility Using Pulsed Laser Deposition. J Material Sci Eng 6: 364. doi: 10.4172/2169-0022.1000364

35. Kudin KN, Ozbas B, Schniepp HC, Prud'homme RK, Aksay IA, et al. (2008) Raman spectra of graphite oxide and functionalized graphene sheets. Nano letters 8: 36-41.

36. Muchharla B, Narayanan TN, Balakrishnan K, Ajayan PM, Talapatra S, et al. (2014) Temperature dependent electrical transport of disordered reduced graphene oxide. 2D Materials 1: 11008.

37. Eda G, Lin YY, Mattevi C, Yamaguchi H, Chen HA, et al. (2010) Blue Photoluminescence from Chemically Derived Graphene Oxide. Advanced Materials 22: 505-509.

38. Lee SJ, Ketterson JB, Trivedi N (1992) Metal-insulator transition in quasi-twodimensional Mo-C films. Physical Review B 46: 12695-12700.

39. Efros AL, Shklovskii BI (1975) Coulomb gap and low temperature conductivity of disordered systems. Journal of Physics C: Solid State Physics 8: 49-51.

40. Peters EC, Giesbers AJM, Burghard M (2012) Variable range hopping in graphene antidot lattices. physica status solidi B 249: 2522-2525.

41. Joung D, Zhai L, Khondaker SI (2011) Coulomb blockade and hopping conduction in graphene quantum dots array. Physical Review B 83: 115323.

42. Joung D, Chunder A, Zhai L, Khondaker SI (2010) Space charge limited conduction with exponential trap distribution in reduced graphene oxide sheets. Applied Physics Letters 97: 93105.

43. Eda G, Mattevi C, Yamaguchi H, Kim H, Chhowalla M (2009) Insulator to Semimetal Transition in Graphene Oxide. The Journal of Physical Chemistry C 113: 15768-15771.
44. Ci L, Song L, Jin C, Jariwala D, Wu D, et al. (2010) Atomic layers of hybridized boron nitride and graphene domains. Nature Materials 9: 430-435.

45. Pearson GL, Bardeen J (1949) Electrical Properties of Pure Silicon and Silicon Alloys Containing Boron and Phosphorus. Physical Review 75: 865-883.

46. Tsang WT, Schubert EF, Cunningham JE (1992) Doping in semiconductors with variable activation energy. Applied Physics Letters 60: 115.

47. Erickson K, Erni R, Lee Z, Alem N, Gannett W, et al. (2010) Determination of the local chemical structure of graphene oxide and reduced graphene oxide. Advanced materials (Deerfield Beach, Fla.) 22: 4467-4472.

48. Gómez-Navarro C, Meyer JC, Sundaram RS, Chuvilin A, Kurasch S, et al (2010) Atomic Structure of Reduced Graphene Oxide. Nano Letters 10: 1144-1148.

49. Adschiri T, Hakuta Y, Sue K, Arai K (2001) Hydrothermal Synthesis of Metal Oxide Nanoparticles at Supercritical Conditions. Journal of Nanoparticle Research 3: 227-235.

50. An X, Simmons T, Shah R, Wolfe C, Lewis KM, et al. (2010) Stable Aqueous Dispersions of Noncovalently Functionalized Graphene from Graphite and their Multifunctional High-Performance Applications. Nano Letters 10: 4295-4301.

51. Bhaumik A, Narayan J (2016) Wafer scale integration of reduced graphene oxide by novel laser processing at room temperature in air. Journal of Applied Physics 120: 105304. 